\title{
Estimating the Cost of Immunoglobulin Replacement Therapy in Primary Immunodeficiency Patients
}

\author{
Hosein Shabaninejad, ${ }^{1}$ Asra Asgharzadeh, ${ }^{2,3,}{ }^{*}$ Nima Rezaei, ${ }^{4,5,6}$ and Aziz Rezapour ${ }^{7,8}$ \\ ${ }^{1}$ Department of Health Management, School of Health Management and Information Sciences, Iran University of Medical Sciences, Tehran, Iran \\ ${ }^{2}$ School of Health Management \& Information Sciences, Iran University of Medical Sciences, Tehran, Iran \\ ${ }^{3}$ Health Technology Assessment Group (HTAG), Universal Scientific Education and Research Network (USERN), Tehran, Iran \\ ${ }^{4}$ Research Center for Immunodeficiencies, Children's Medical Center, Tehran University of Medical Sciences, Tehran, Iran \\ ${ }^{5}$ Department of Immunology and Biology, School of Medicine, Tehran University of Medical Sciences, Tehran, Iran \\ ${ }^{6}$ Network of Immunity in Infection, Malignancy and Autoimmunity (NIIMA), Universal Scientific Education and Research Network (USERN), Tehran, Iran \\ ${ }^{7}$ Health Management and Economics Research Center, Iran University of Medical Sciences, Tehran, Iran \\ ${ }^{8}$ Department of Health Economics, School of Health Management and Information Sciences, Iran University of Medical Sciences, Tehran, Iran \\ "Corresponding author: Asra Asgharzadeh, School of Health Management and Information Sciences, Iran University of Medical Sciences, Tehran, Iran. Tel: +98-2161472121, \\ E-mail: as.asgharzadeh@gmail.com
}

Received 2017 April 09; Accepted 2017 June 30.

\begin{abstract}
Background: Immunoglobulin (Ig) replacement therapy is used for treating a variety of primary immunodeficiency diseases (PIDs). Ig replacement therapy is the most important therapy for these diseases, since it protects the body against infections and reduces autoimmune disease symptoms. The purpose of this research was to estimate the cost of subcutaneous and intravenous Ig therapy in Iran.

Methods: This study is carried out from the perspective of Iran's healthcare system and all the medical costs are calculated. Cost variables include personnel, equipment, and supplies. The data required for cost estimation are obtained from the records of children's Medical center of Tehran for 2015. Personnel and medical treatment costs are calculated based on relative value units. For SCIg, cost items are extracted from the literature.

Results: The total cost of IVIg and SCIg for the health system in the first year of treatment is $\$ 1370$ and $\$ 121$ respectively. The results indicate that SCIg reduces costs and is the preferred treatment for PID patients.

Conclusions: SCIg therapy significantly reduces the costs of the healthcare system compared to IVIg therapy, and this is enough economic justification for introduction of this treatment in the Iranian healthcare system. SCIg is also critical in reducing the direct costs of patients.
\end{abstract}

Keywords: Cost Analysis, Primary Immunodeficiency, Immunoglobulin Replacement, Healthcare System

\section{Background}

Rapid advances in immunology in recent decades have increased our understanding of primary immunodeficiency diseases (PIDs) (1). PIDs are caused by genetic or developmental defects in the immune system or by an absent or non-functional gene product critical for the normal immune function. These diseases are characterized by severe complications and early mortality (2). According to statistics, 25 in 1 million people are born with a PID $(3,4)$. In Iran, there are 1,661 recorded PID patients in the Children's Medical Center of Tehran. Due to the prevalence of consanguinous marriages, the incidence of autosomal-dominant PIDs is very high. Moreover, about 70 - 90 percent of patients may die before they are diagnosed (5).

Early diagnosis and treatment of PIDs is crucial to the longevity of the patients. Immunoglobulin (Ig) replacement therapy is an important treatment for a variety of conditions in the fields of dermatology, neurology, hematology, and immunology (6). For the majority of PID pa- tients it is the only life-saving therapy and treatment is lifelong, since the vast majority of them have primary antibody failure. Successful treatment depends on factors such as availability of products, the type of immunodeficiency and any comorbidities of the individual patient. Essential components include long-term follow-up, regular monitoring and a close relationship between the patient and the multidisciplinary clinical immunology team (7).

In intravenous immunoglobulin therapy (IVIg), Ig directly enters the blood stream. The main advantage of this method is that high Ig doses can be administered via each infusion, and doses are usually given every three to four weeks (8). On the other hand, infusions must be done in a hospital or at home by a nurse or a trained individual. Also patients may experience a reaction during or after infusion (9). Ig products are somewhat different and may be tolerated differently by patients (10).

Subcutaneous immunoglobulin (SCIg) is administered using a syringe or pump. Common infusion sites 
include the abdomen, upper arms, or hips. Infusion time varies from one to two hours and the weekly dose can be divided and delivered in several days. The patient or their family can be trained to administer SCIg themselves (11). The present research tries to estimate the costs of Ig replacement therapy in PID patients using IVIg and SCIg.

\section{Methods}

\subsection{Costing}

This study is carried out from the perspective of healthcare providers and all the cost items are identified and analyzed based on the treatment protocols for PID patients. Data are collected from the children's medical center of Tehran.

Medical costs are determined using direct costing and consultation with experts. To analyze all the direct costs associated with each intervention, the literature was reviewed, standards were identified, and focus groups of immunology professions were settled to examine all the processes, resources, and activities involved in preparation and provision of services to patients.

\subsection{Cost Variables}

Cost variables include personnel, equipment, and medical supplies. Table 1 shows the cost items and their estimation.

The cost of medical supplies is calculated for each treatment session. Due to similar Ig doses in IVIg and SCIg, the cost of Ig products is not taken into account. Staff wages as well as the costs related to inpatient bed and medical supplies are calculated based on the fees set by the ministry of health.

Inpatient bed cost is the highest equipment cost, which is the cost of one-day stay. The annual depreciation of each hospital bed equals $7 \%$ of the cost of building each bed, while the cost of depreciation per active hospital bed is calculated as the annual depreciation of beds in 2015; depreciation per active hospital bed $=365 \times$ percentage of occupied beds in hospitals affiliated with Tehran University of Medical Sciences.

\subsection{Cost Analysis}

Given the focus of the study, the intangible costs of patients and their families such as stress, discomfort, and pain are not calculated. Since the period of study is one year, discount rate is not taken into consideration. Also the costs are converted to US dollars (1 USD equals 30000 Iranian Rials).

\subsection{Sensitivity Analysis}

Due to uncertainty and changes in resources used, sensitivity analysis was performed based on the number of visits per year (IVIg infusion is done every 3 - 4 weeks while SCIg infusion is done once a week), personnel, equipment and supplies (10\% decrease for each item).

\section{Results}

IVIg infusions are generally administered once every three or four weeks and treatment is lifelong (12 - 16 sessions annually). The number of annual SCIg infusions is considered to be four times that of IVIg. The annual IVIg treatment cost consists of the monthly costs of patients plus the healthcare resources used for diagnosis and treatment. Cost items associated with SCIg are extracted from foreign studies and adjustments are made based on experts' views. It must be noted that SCIg is an infusion method that administers $600 \mathrm{mg} / \mathrm{kg}$ dose of $\mathrm{Ig}$ in four parts, maintaining the same Ig level of IVIg method administered once monthly.

In order to switch from IVIg to SCIg, patients are trained to self-administer the procedure and are monitored weekly by local hospital care (12). Usually a 4 - 6 session training course is required for home-based infusion preparation by the patients or their family. Medical and nursing follow-up care and support is offered during training (13). SCIg requires a nurse for patient training (6 hours during the first year) and for annual follow-up (6 hours). IVIg requires a nurse, a unit clerk, a scheduling clerk, and a ward aid (14). Table 2 provides the unit cost of Ig replacement service in IVIg and SCIg based on the aforementioned assumptions.

Table 3 provides the total cost of Ig replacement using IVIg and SCIg during the first year of treatment. The data show that the total cost is $\$ 1370$ for IVIg and 121 for SCIg. Moreover, the total cost of each item is also calculated. The results indicate that equipment and supplies are the most expensive costs both in IVIg and SCIg.

\subsection{Sensitivity Analysis}

In this part, we estimate the effect of changes in each cost variable (i.e. equipment, personnel, and supplies) (Table 4). In all scenarios, every condition for SCIg is calculated as 4 times that of IVIg (12 annual IVIg sessions versus 48 annual SCIg sessions).

The results of sensitivity analysis show that changes in the number of infusion sessions affect the annual cost of treatment, while changes in cost items have little effect on the final treatment cost. Reduction in the cost of equipment has the greatest effect on total costs. 
Table 1. Cost Items and Their Estimation

\begin{tabular}{|c|c|c|}
\hline Variable & Cost Item & Description \\
\hline \multirow{3}{*}{ Supplies } & $\operatorname{Ig}(g)$ & Ig dose is similar in both IVIg and SCIg \\
\hline & Laboratory costs & \\
\hline & $\begin{array}{l}\text { Dextrose/saline, adhesive tape, adhesive bandage, alcohol } \\
\text { swabs, gauze, infusion set }\end{array}$ & Medical supplies used in each treatment session \\
\hline \multirow{7}{*}{ Personnel } & Outpatient visit by a specialist & Fixed fees \\
\hline & Inpatient visit & Fixed fees \\
\hline & Intravenous infusion by a specialist & Fixed fees \\
\hline & Average nurse wage for each session & $\begin{array}{l}6 \text { hours per session (assuming a minimum monthly salary of } \$ 600 \text { for } 176 \\
\text { working hours) }\end{array}$ \\
\hline & Check-up visit & An appointment with the doctor every six months (twice a year) \\
\hline & Training by a nurse & 6 hours during the first year $(30$ minutes minimum $=\$ 3.50)$ \\
\hline & Annual follow-up by a nurse & 6 hours (assuming a minimum monthly salary of $\$ 600$ for 176 working hours) \\
\hline \multirow{5}{*}{ Equipment } & Inpatient bed cost & The cost of one-day stay \\
\hline & Annual depreciation of hospital beds* & Equivalent to 7\% of the cost of building each hospital bed in 2015 \\
\hline & Inpatient bed occupancy rate & Tehran, 2015 \\
\hline & Average construction cost per bed & Tehran, 2015 \\
\hline & The cost of depreciation per active hospital bed & $\begin{array}{l}\text { Annual depreciation of beds in } 2015 /(365 \times \text { percentage of occupied beds in the } \\
\text { hospitals affiliated with Tehran University of Medical Sciences, 2015) }\end{array}$ \\
\hline
\end{tabular}

Table 2. Unit Cost of Immunoglobulin Replacement Therapy Through Subcutaneous and Intravenous Infusion

\begin{tabular}{|c|c|c|}
\hline \multirow[t]{2}{*}{ Item } & \multicolumn{2}{|c|}{ Cost, $\mathbf{S}$} \\
\hline & IVIg & SCIg \\
\hline \multicolumn{3}{|l|}{ Hospital costs } \\
\hline Inpatient bed cost & 35.88 & - \\
\hline Depreciation of active hospital beds* & 31.77 & - \\
\hline \multicolumn{3}{|l|}{ Personnel costs } \\
\hline Outpatient visit by a specialist & 3.81 & - \\
\hline Inpatient visit & 13.41 & - \\
\hline Intravenous infusion by a specialist & 0.58 & - \\
\hline Nursing and other services & 20.47 & - \\
\hline Nursing follow-up & - & 20.47 \\
\hline Training by a nurse & 41.98 & 41.98 \\
\hline \multicolumn{3}{|l|}{ Drugs and medical supplies } \\
\hline $\operatorname{Ig}(\mathrm{g})$ & - & - \\
\hline Laboratory costs & 0.73 & 0.73 \\
\hline Medical supplies & 4.47 & 0.50 \\
\hline Unit cost of treatment & 153.11 & 63.68 \\
\hline
\end{tabular}

\section{Discussion}

In this study, we determined cost items of IVIg and SCIg based on expert opinions and literature review. The reIran J Pediatr. 2017; 27(4):e6294. sults show supplies, equipment and personnel consist 5, 36 and 59 percent of total costs, respectively. Moreover, sensitivity analysis depicts that changes in the number of administration sessions of the two methods are more effective than changes in cost items. Based on the present cost estimation, hospital costs (personnel and inpatient beds) are the most expensive costs associated with IVIg, while these costs can be avoided by switching to SCIg. Generally, cost estimation of Ig replacement in the Children's medical center of Tehran shows that IVIg is more costly than SCIg and switching from IVIg to SCIg is a valuable option for reducing treatment costs (\$1370 vs. \$121).

Ho et al. reported similar results and showed that hospital-based IVIg (\$21.27) is more costly than home-based IVIg (\$19.43) and SCIg (\$20.06). Overall, the results of the study showed that SCIg is more cost-effective than hospitalbased IVIg (15). In another study, Liu et al. reported the lower cost of SCIg compared to IVIg (16). Also Abolhassani et al. showed that switching from IVIg to SCIg leads to $25 \%$ $33 \%$ reduction in costs (17). Similarly, Beaute et al. reported a $25 \%$ reduction in costs as a result of switching to $\mathrm{SCIg}(18)$. Martin et al. conducted a study in Canada and found a significant cost difference between IVIg and SCIg and a 74\% reduction in costs by switching from IVIg to SCIg mainly due to less personnel employment (14). A study in the US reported similar findings and attributed the lower cost of 
Table 3. Estimating the Cost of Immunoglobulin Replacement Therapy Through Subcutaneous and Intravenous by Cost Items

\begin{tabular}{|c|c|c|c|c|}
\hline \multirow[t]{2}{*}{ Cost Item } & \multicolumn{2}{|c|}{ IVIg } & \multicolumn{2}{|c|}{ SCIg } \\
\hline & Cost (USD), $S$ & \% Total cost & Cost (USD), $S$ & \% Total cost \\
\hline Supplies & 62 & 5 & 59 & 53 \\
\hline Personnel & 500 & 36 & 62 & 47 \\
\hline Equipment & 809 & 59 & 0 & 0 \\
\hline Total & 1370 & 100 & 121 & 100 \\
\hline
\end{tabular}

Table 4. Sensitivity Analysis

\begin{tabular}{lcccc}
\hline Scenarios & 12 IVIg Sessions, S & 48 SCIg Sessions, \$ & 16 IVIg Sessions, \$ & 1813 \\
\hline$A^{\text {a }}$ & 1370 & 121 & 1748 \\
$\mathbf{B}^{\mathbf{b}}$ & 1320 & 115 & 140 \\
$\mathbf{C}^{\mathbf{C}}$ & 1290 & 121 & 1705 & 134 \\
$\mathbf{D}^{\mathbf{d}}$ & 1364 & 115 & 1805 & 130 \\
\hline
\end{tabular}

${ }^{\text {a }}$ Scenario A: Fixed equipment, personnel, and medical supply costs.

${ }^{\mathrm{b}}$ Scenario B: $10 \%$ reduction in personnel costs.

${ }^{\mathrm{c} S}$ Scenario C: $10 \%$ reduction in equipment costs.

${ }^{\mathrm{d}}$ Scenario D: $10 \%$ reduction in the cost of medical supplies.

SCIg to higher hospital costs in IVIg treatment compared to infusion pumps used in SCIg (16). Igarashi et al. (2014) evaluated the cost differences between IVIg and SCIg and showed that reduced hospital visits is the main reason for the economic benefits of home-based SCIg (19).

The present study has few limitations. The costs of interventions were calculated from the perspective of healthcare providers and it was impossible to calculate the total cost of healthcare services. Therefore, the relative value of health services was used in cost estimation. Moreover, since it was impossible to follow up patients in different periods of the one-year time horizon, and as a result discount rate was not applied. Therefore, the results have limited generalizability and must be considered with caution.

Summed up, the present findings show that switching from IVIg to SCIg can reduce the costs of Iran's healthcare system and, supported by evidence from other countries, there is enough economic justification to adopt SCIg in this country. It is thus recommended to use this emerging technology for those patients that require treatment with fewer costs and as a complementary treatment for others. Patients must be given the chance to choose between SCIg and IVIg and those who opt for SCIg must be provided with effective training and support.

\subsection{Conclusion}

The results showed that the total cost of IVIg and SCIg for the first year of treatment is $\$ 1370$ and $\$ 121$ respectively.
This is supported by various studies in different countries, all of which suggest that SCIg is less costly than IVIg and that SCIg is a more cost-effective treatment (20). Switching from IVIg to SCIg can significantly reduce the direct costs for patients.

Investment in the implementation and promotion of SCIg is of vital importance. SCIg can be administered at home, thus reducing the frequency of hospital visits and bed occupancy. As a result, it plays a crucial role in reducing the costs of the healthcare system and saving resources.

Overall, advances in immunology and the ongoing attempt to reduce the costs of treatments and improve patients' quality of life is expected to increase the use of SCIg over IVIg in Iran as a less costly and equally effective treatment. To expand the use of this new treatment, increased cooperation with the government is needed to provide the necessary conditions for PID patients, including training courses, annual follow-up, and support. Thus, SCIg will be more accessible and less costly for patients who are willing to use SCIg or are looking for a more cost-effective treatment than IVIg. It must be noted that proper use of new technologies in renowned medical centers can increase public trust in them. 


\section{Acknowledgments}

The authors would like to express their sincere thanks to the neumerous reviewers for their valuable help. We would like to extent our gratitude to Dr Hassan Abolhassani and Mr Mohammadreza Mobinizadeh for the guidance and encouraging us to compose this article. (Both of whom contributed to the costs data extraction and data analysis). This work was supported by a grant (IUMS/SHMIS-2015/7) from Iran University of Medical Sciences.

\section{Footnotes}

Declaration of Interests: The authors report no conflicts of interest. The authors alone are responsible for the content and writing of the paper.

Ethical Issues: The ethics committee of Iran University of Medical Sciences has approved our study.

Implications for Policy Makers: Administration of SCIg at home plays a crucial role in reducing the costs of the healthcare system and saving resources via reducing the frequency of hospital visits and bed occupancy. SCIg as a new method of treatment improves quality of life of patients with immunodeficiency, with the same or more effectiveness and less cost. Switching from IVIg to SCIg reduces the costs of Iran's healthcare system and, supported by evidence from other countries, there is enough economic justification to adopt SCIg in Iran.

Implications for Public: Considering advances in immunology with ongoing attempt to reduce the costs of treatments and improve patients' quality of life, it is expected to increase the use of SCIg over IVIg in Iran as a less costly and more effective treatment method. Thus, SCIg will be more accessible and less costly for patients who are willing to use SCIg instead of IVIg. Our study recommends using this emerging technology for those patients that require an affordable treatment and as a complementary treatment for others. Patients should have a chance to choose between SCIg and IVIg, and those who opt SCIg should be provided with effective training and support.

\section{References}

1. Bousfiha A, Picard C, Boisson-Dupuis S, Zhang SY, Bustamante J, Puel A, et al. Primary immunodeficiencies of protective immunity to primary infections. Clin Immunol. 2010;135(2):204-9. doi: 10.1016/j.clim.2010.02.001. [PubMed: 20236864].

2. Aghamohammadi A, Moin M, Rezaei N. History of primary immunodeficiency diseases in iran. Iran J Pediatr. 2010;20(1):16-34. [PubMed: 23056678].
3. Borte S, Wang N, Oskarsdottir S, von Dobeln U, Hammarstrom L. Newborn screening for primary immunodeficiencies: beyond SCID and XLA. Ann N Y Acad Sci. 2011;1246:118-30. doi: 10.1111/j.17496632.2011.06350.x. [PubMed: 22236436].

4. Puck JM. Neonatal screening for severe combined immunodeficiency. Curr Opin Pediatr. 2011;23(6):667-73. doi: 10.1097/MOP.ob013e32834cb9bo. [PubMed: 22001765].

5. Aghamohammadi A, Mohammadinejad P, Abolhassani H, Mirminachi B, Movahedi M, Gharagozlou M, et al. Primary immunodeficiency disorders in Iran: update and new insights from the third report of the national registry. J Clin Immunol. 2014;34(4):478-90. doi: 10.1007/s10875-014-0001-z. [PubMed: 24659230].

6. Rezaei N, Abolhassani H, Aghamohammadi A, Ochs HD. Indications and safety of intravenous and subcutaneous immunoglobulin therapy. Expert Rev Clin Immunol. 2011;7(3):301-16. doi: 10.1586/eci.10.104. [PubMed: 21595597].

7. Peter JG, Chapel H. Immunoglobulin replacement therapy for primary immunodeficiencies. Immunotherapy. 2014;6(7):853-69. doi: 10.2217/imt.14.54. [PubMed: 25290417].

8. Lucas M, Lee M, Lortan J, Lopez-Granados E, Misbah S, Chapel H. Infection outcomes in patients with common variable immunodeficiency disorders: relationship to immunoglobulin therapy over 22 years. J Allergy Clin Immunol. 2010;125(6):1354-1360 e4. doi: 10.1016/j.jaci.2010.02.040. [PubMed: 20471071].

9. Shapiro RS. Why I use subcutaneous immunoglobulin (SCIG). J Clin Immunol. 2013;33 Suppl 2:S95-8. doi: 10.1007/s10875-012-9853-2. [PubMed: 23264027].

10. Schroeder HJ, Dougherty CJ. Review of intravenous immunoglobulin replacement therapy trials for primary humoral immunodeficiency patients. Infection. 2012;40(6):601-11. doi: 10.1007/s15010-012-0323-9. [PubMed: 22968971].

11. Soler-Palacin P, Gaso-Gago I, Fernandez-Polo A, Martin-Nalda A, Oliveras $M$, Martinez-Cutillas J, et al. Intravenous and subcutaneous immunoglobulin replacement: a two-way road. Optimizing healthcare quality in patients with primary immunodeficiencies. J Clin Immunol. 2014;34(8):1015-7. doi:10.1007/s10875-014-0096-2. [PubMed: 25190197].

12. Vultaggio A, Azzari C, Milito C, Finocchi A, Toppino C, Spadaro G, et al. Subcutaneous immunoglobulin replacement therapy in patients with primary immunodeficiency in routine clinical practice: the VISPO prospective multicenter study. Clin Drug Investig. 2015;35(3):179-85. doi:10.1007/s40261-015-0270-1. [PubMed: 25672929].

13. Membe SK, Ho C, Cimon K, Morrison A, Kanani A, Roifman CM. Economic assessment of different modalities of immunoglobulin replacement therapy. Immunol Allergy Clin North Am. 2008;28(4):861-74. doi: 10.1016/j.iac.2008.06.008. [PubMed: 18940579] x.

14. Martin A, Lavoie L, Goetghebeur M, Schellenberg R. Economic benefit of subcutaneous rapid push versus intravenous immunoglobulin infusion therapy in adult patients with primary immune deficiency. Allergy Asthma Clin Immunol. 2012;8(Suppl 1):20. doi:10.1186/1710-14928-s1-a20.

15. Ho C, Membe SK, Cimon K. An overview of subcutaneous vs. intravenous immunoglobulin for primary immunodeficiencies: systematic review and economic analysis. Canadian Agen Drugs Technol Health. 2008;36:1-8.

16. Liu Z, Albon E, Hyde C. The effectiveness and cost effectiveness of immunoglobulin replacement therapy for primary immunodeficiency and chronic lymphocytic leukaemia: a systematic review and economic evaluation. University of Birmingham, Department of Public Health and Epidemiology; 2005.

17. Abolhassani H, Sadaghiani MS, Aghamohammadi A, Ochs HD, Rezaei N. Home-based subcutaneous immunoglobulin versus hospitalbased intravenous immunoglobulin in treatment of primary antibody deficiencies: systematic review and meta analysis. J Clin Immunol. 2012;32(6):1180-92. doi: 10.1007/s10875-012-9720-1. [PubMed: 22730009]. 
18. Beaute J, Levy P, Millet V, Debre M, Dudoit Y, Le Mignot L, et al. Economic evaluation of immunoglobulin replacement in patients with primary antibody deficiencies. Clin Exp Immunol. 2010;160(2):240-5. doi: 10.1111/j.1365-2249.2009.04079.x. [PubMed: 20041884].

19. Igarashi A, Kanegane H, Kobayashi M, Miyawaki T, Tsutani K. Costminimization analysis of IgPro20, a subcutaneous immunoglobulin, in Japanese patients with primary immunodeficiency. Clin Ther. 2014;36(11):1616-24. doi: 10.1016/j.clinthera.2014.08.007. [PubMed:
25236916].

20. Shabaninejad H, Asgharzadeh A, Rezaei N, Rezapoor A. A Comparative Study of Intravenous Immunoglobulin and Subcutaneous Immunoglobulin in Adult Patients with Primary Immunodeficiency Diseases: A Systematic Review and Meta-Analysis. Expert Rev Clin Immunol. 2016;12(5):595-602. doi: 10.1586/1744666X.2016.1155452. [PubMed: 26902306]. 